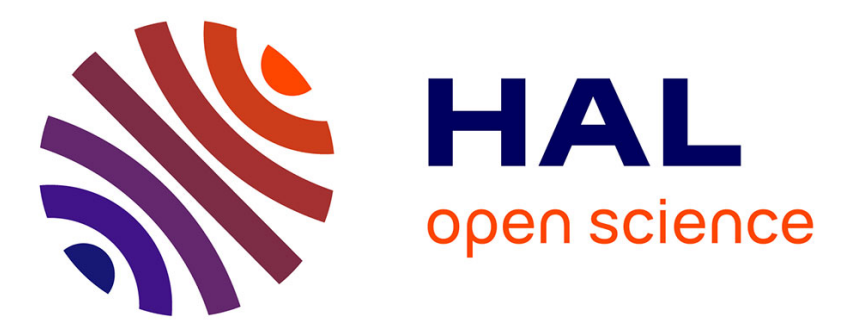

\title{
Improved finite difference method for equilibrium problems based on differentiation of the partial differential equations and the boundary conditions
}

\author{
M. Arad, R. Segev, G. Ben-Dor
}

\section{- To cite this version:}

M. Arad, R. Segev, G. Ben-Dor. Improved finite difference method for equilibrium problems based on differentiation of the partial differential equations and the boundary conditions. International Journal for Numerical Methods in Engineering, 1995, 38 (11), pp.1831-1853. hal-01068219

HAL Id: hal-01068219

https://hal.science/hal-01068219

Submitted on 25 Sep 2014

HAL is a multi-disciplinary open access archive for the deposit and dissemination of scientific research documents, whether they are published or not. The documents may come from teaching and research institutions in France or abroad, or from public or private research centers.
L'archive ouverte pluridisciplinaire HAL, est destinée au dépôt et à la diffusion de documents scientifiques de niveau recherche, publiés ou non, émanant des établissements d'enseignement et de recherche français ou étrangers, des laboratoires publics ou privés. 


\title{
IMPROVED FINITE DIFFERENCE METHOD FOR EQUILIBRIUM PROBLEMS BASED ON DIFFERENTIATION OF THE PARTIAL DIFFERENTIAL EQUATIONS AND THE BOUNDARY CONDITIONS
}

\author{
M. ARAD, R. SEGEV AND G. BEN-DOR \\ Pearlstone Center for Aeronautical Engineering Studies, Department of Mechanical Engineering, \\ Ben-Gurion University of the Negev, Beer Sheva, Israel
}

\section{SUMMARY}

A numerical algorithm for producing high-order solutions for equilibrium problems is presented. The approximated solutions are improved by differentiating both the governing partial differential equations and their boundary conditions.i.

The advantages of the proposed method over standard finite difference methods are: the possibility of using arbitrary meshes; the possibility of using simultaneously approximations with different (distinct) orders of accuracy at different locations in the problem domain; an improvement in approximating the boundary conditions; the elimination of the need for 'fictitious' or 'external' nodal points in treating the boundary conditions.

Furthermore, the proposed method is capable of reaching approximate solutions which are more accurate than other finite difference methods, when the same number of nodal points participate in the local scheme.

A computer program was written for solving two-dimensional problems in elasticity. The solutions of a few examples clearly illustrate these advantages.

KEY WORDS: numerical methods; finite differences; plane elasticity

\section{INTRODUCTION}

The rapid growth in the use of computers in the past decade gave rise to the development of advanced numerical methods. The two basic ones are the finite element and the finite difference methods. The advantages of the finite element method over the finite difference method were presented by Zienkiewicz. ${ }^{1}$ They, are:

(a) the ease of arbitrary positioning of nodal points;

(b) the infinite possibilities of generation of 'improved' elements by simply increasing the number of element parameters; :

(c) the improvement in the boundary value approximation due to its integral form; and

(d) the ease with which different types and sizes of elements can be adopted.

Since the pioneering work of Southwell ${ }^{2,3}$ in which the finite difference method was comprehensively formulated, many works; attempting to improve the method, have been published. For example, Forsyth and Wasow 4 isuggested to treat curved boundaries by the use of interpolations between points on the boundariesi and nodal points adjacent to them. Wang ${ }^{5}$ and Collat $z^{6}$ 
treated curved boundaries having Dirichlet conditions by using Taylor series expansions. A different approach for treating curved boundaries, still using a square or rectangular mesh, was forwarded by Varga. ${ }^{7}$ In this approach, sometimes referred to as the control volume approach, an integration is performed over the mesh areas using Green's theorem. This approach has been improved and applied in many areas e.g. by Griffin and Kellogg, ${ }^{8}$ Utka, ${ }^{9}$ Havner and Stanton, ${ }^{10}$ Havner, ${ }^{11}$ Johnson $^{12}$ and others.

As shown by Jensen ${ }^{13}$ one of the difficulties arising from the use of a square or rectangular mesh with curved boundaries is the need to increase the number of the nodes over the number actually necessary to achieve a particular accuracy in the solution. One way of overcoming this shortcoming is the use of a triangle mesh. Triangle mesh was probably first proposed by MacNeal ${ }^{14}$ who investigated current flows through thin sheets. Application of triangle mesh which uses finite differences derived from the variational approach and Ritz method appeared in the works of Kellogg and his coworkers. ${ }^{15-18} \mathrm{~A}$ different approach for using triangular mesh originally proposed by Winslow ${ }^{19}$ was generalized by Chu. ${ }^{20,21}$ The idea was to replace the rectangular (or square) mesh with an equilateral triangle mesh plane. The replacement involved an 'oblique transformation' of the entire domain. A somewhat different approach, which in fact is an improvement, was presented by Frey ${ }^{22}$ who used the concept of isoparametric elements. $\mathrm{He}$ introduced flexible finite difference stencils of arbitrary shape mapped into a regular square mesh.

Jensen ${ }^{13}$ proposed a different approach for generating finite difference approximations in an arbitrary mesh by using two-dimensional Taylor series expansions. He showed that by using this approach, the error in the approximated Taylor series expansions converges to zero when the nodes density is increased. Second-order expansions were used by him on a six-point control scheme (template). The main disadvantage of his approach was frequent singularity or illconditioning of the six-point control scheme. More details regarding this singularity are given subsequently. Several authors tried to develop an automatic procedure which avoids incorrect schemes and thus improves the accuracy of the finite difference formula. Perrone and $\mathrm{Kao}^{23}$ suggested that additional nodes in the six-point scheme should be considered and an averaging process for the generation of finite difference coefficients applied. Liszka and Orkisz ${ }^{24}$ proposed to increase the number of the neighbouring nodes in the scheme forming an over-determined set of linear equations. Their solution was obtained by a minimization of a norm that they derived. In this way a set of five equations with five unknowns was obtained. Noye and Arnold ${ }^{25}$ developed a more accurate finite difference scheme for approximating the derivatives at points that are near a curved boundary along which a Neumann boundary condition applies. There is no need to reshape the curved boundary, and the approximations at adjacent grid points have the same accuracy as schemes commonly used for Dirichlet boundary conditions. Dow et al. ${ }^{26}$ improved the boundary modelling for finite difference applications in solid mechanics. The improvement was expressed by introducing the physical nature of the deformations into the equations by which the solution is approximated. This is achieved by expressing the coefficients of the Taylor series expansions which approximate the displacements in terms of rigid body motions, strains and derivatives of strains. As a result the fictitious nodal points were rationally incorporated into the mesh. This approach enabled one to use an arbitrary mesh provided the problem domain was rectangular. In their example they used Taylor series expansions with nine coefficients using Pascal's triangle, i.e. second-order Taylor expansions with three additional coefficients (note that this is identical to the regular nine-point finite element approximation). In a subsequent modification Dow and Hardaway 27 applied the approach to curved boundaries and concave corners. More details regarding the derivation of the displacement expansions can be found in References 28-31. Kochavi et al. ${ }^{32}$ used non-conforming Taylor discretization that allowed discrepancy between values resulting from the Taylor series expansions about distinct nodes if the discrepancy 
is in the same order of magnitude as the estimated error resulting from the discretization. This method was capable of producing results that were more accurate than those obtained by traditional conforming discretizations. In addition, unnecessary computations might be avoided by adjusting the accuracy of the solution of the algebraic equations to the truncation error in the Taylor series expansions.

The main disadvantage of all these studies is that they approximate the solutions by second-order Taylor series expansions in which the error is of the third order, i.e. $O\left(h^{3}\right)$ where $h$ is the grid size. Thus, the errors associated with the approximations of the second-order derivatives in an arbitrary mesh (which usually appear in the conservation equations) are very large, as they are of the order of $O(h)$.

In general, Jensen ${ }^{13}$ showed that with this formulation the derivatives of order $i$ can be calculated with an accuracy $O\left(h^{m+1-i}\right)$, by using $N_{n}=\frac{1}{2}(m+1)(m+2)$ neighbouring values $(m$ is the order of the Taylor series expansions). For example, the difference expressions for all derivatives up to the fourth order require at least $N_{n}=15$ neighbouring values and consequently the inversion of a $15 \times 15$ nodal matrix for each point! In view of the computational effort required to invert a $15 \times 15$ matrix it is appropriate to look for ways of alleviating this problem.

The new method which will be presented in the next section has all the advantages associated with the finite element method as introduced by Zienkiewicz.' Furthermore, in the proposed method, approximations using Taylor series expansions of the $m$ th order require at most $N_{\mathrm{n}}=2 m+2$ neighbouring values, instead of the above-mentioned requirement of $N_{\mathrm{n}}=\frac{1}{2}(m+1)(m+2)$ neighbouring values.

\section{THE PROPOSED METHOD}

The proposed method which is applicable to equilibrium problems is an improved Differentiation Based Finite Difference Method (DFD-method). In the DFD-method both the governing equations and their boundary conditions are differentiated. The model is a mathematical one and independent of a specific physical problem. In order to simplify its introduction, it will be applied to problems in elasticity. However, it should be emphasized here that it could be applied to equilibrium problems from other fields e.g. steady viscous flows, etc.

\section{INTRODUCTION OF THE PROBLEM TO BE SOLVED}

In the following, the DFD-method will be illustrated by applying it to the solution of the equilibrium equations in terms of displacements $(u$ and $v$ ) in plane elasticity (for details see p. 265 of Reference 33). The equilibrium equations are

$$
\left.\begin{array}{l}
-\frac{\partial}{\partial x}\left(c_{11} \frac{\partial u}{\partial x}+c_{12} \frac{\partial v}{\partial y}\right)-c_{33} \frac{\partial}{\partial y}\left(\frac{\partial u}{\partial y}+\frac{\partial v}{\partial x}\right)=f_{x} \\
-c_{33} \frac{\partial}{\partial x}\left(\frac{\partial u}{\partial y}+\frac{\partial v}{\partial x}\right)-\frac{\partial}{\partial y}\left(c_{12} \frac{\partial u}{\partial x}+c_{22} \frac{\partial v}{\partial y}\right)=f_{y}
\end{array}\right\} \text { in } \Omega
$$

the boundary conditions can be either primary (displacements)

$$
\left.\begin{array}{l}
u=\tilde{u} \\
v=\tilde{v}
\end{array}\right\} \quad \text { on } \Gamma_{1}
$$


or natural (traction)

$$
\left.\begin{array}{l}
\left(c_{11} \frac{\partial u}{\partial x}+c_{12} \frac{\partial v}{\partial y}\right) n_{x}+c_{33}\left(\frac{\partial u}{\partial y}+\frac{\partial v}{\partial x}\right) n_{y}=\tau_{x} \\
c_{33}\left(\frac{\partial u}{\partial y}+\frac{\partial v}{\partial x}\right) n_{x}+\left(c_{12} \frac{\partial u}{\partial x}+c_{22} \frac{\partial v}{\partial y}\right) n_{y}=\tilde{t}_{y}
\end{array}\right\} \text { on } \Gamma_{2}
$$

where $u$ and $v$, the displacements in the $x$ and $y$ directions, respectively, are the unknown variables; $f_{x}$ and $f_{y}$ denote the body forces in the $x$ and $y$ directions, respectively; $n_{x}$ and $n_{y}$ are the $x$ and $y$ components of a unit vector n normal to the boundary $\Gamma_{;} \Gamma_{1}$ and $\Gamma_{2}$ are (disjoints) portions of the boundary $\left(\Gamma_{1}\right.$ and $\Gamma_{2}$ do not overlap except for a small number of discrete points-singular points); $\tilde{t}_{x}$ and $\tilde{t}_{y}$ denote specified boundary (traction) forces in the $x$ and $y$ directions, respectively; $\tilde{u}$ and $\tilde{v}$ are specified displacements in the $x$ and $y$ direction, respectively;

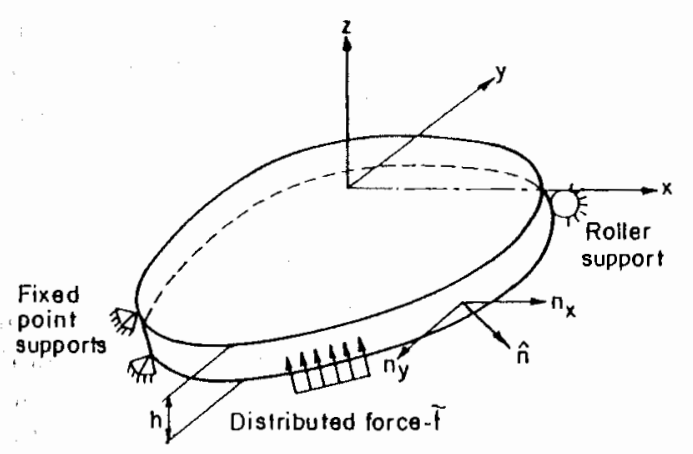

(0)

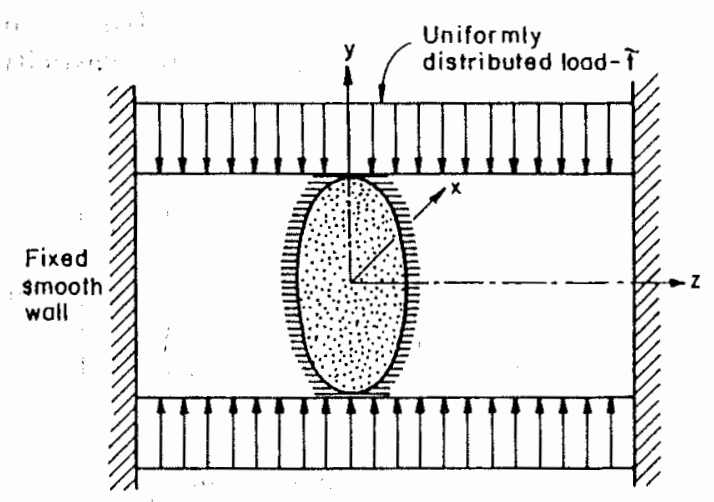

(b)

Figure 1. Schematic illustration of two-dimensional plane elasticity problems. (a) plane stress; (b) plane strain 
$c_{i j}$ are the elasticity (material) constants. For an isotropic elastic body they are given in terms of the modulus of elasticity $E$ and the Poisson's ratio $v$ as follows:

for plane stress

$$
c_{11}=c_{22}=w \frac{E}{1-v^{2}}, \quad c_{12}=w \frac{v E}{1-v^{2}}, \quad c_{33}=w \frac{E}{2(1+v)}
$$

for plane strain

$$
c_{11}=c_{22}=w \frac{E(1-v)}{(1+v)(1-2 v)}, \quad c_{12}=w \frac{E v}{(1+v)(1-2 v)}, \quad c_{33}=w \frac{E}{2(1+v)}
$$

where $w$ is the thickness of the plate. For the reader's convenience the above definitions are shown in Figure 1.

For an isotropic elastic body without body forces, the above equations can be simplified to the following form:

$$
\begin{aligned}
& c_{11} u_{x^{2}}+c_{33} u_{y^{2}}+\left(c_{12}+c_{33}\right) v_{x y}=0 \\
& c_{33} v_{x^{2}}+c_{11} v_{y^{2}}+\left(c_{12}+c_{33}\right) u_{x y}=0
\end{aligned}
$$

where

$$
u_{x^{2}}=\frac{\partial^{2} u}{\partial x^{2}}, \cdot u_{y^{2}}=\frac{\partial^{2} u}{\partial y^{2}}, \quad u_{x y}=\frac{\partial^{2} u}{\partial x \partial y}, \text { etc. }
$$

These derivatives are written in the following condensed form:

$$
u_{x^{a} y^{b}}=\frac{\partial^{a+b}}{\partial x^{a} \partial y^{b}}
$$

In the following the solution of equations (5a) and (5b) will be approximated for the boundary conditions given by equations (2) or (3).

\section{METHOD OF SOLUTION}

The first step of the solution method is to scatter $N$ nodal points in the computation domain and along the boundary as shown in Figure 2. The way of positioning the nodal points in order to avoid singularity and ill-conditioning will be described subsequently. The $i$ th nodal point will be defined by its $x$ and $y$ co-ordinates $\left(x_{i}, y_{i}\right)$. Around any nodal point one can derive Taylor series expansions of any order (e.g. $m$ ) which will approximate the unknown variable (i.e. the displacements $u$ and $v$ in the present example). A similar approach was adopted, for example, by Dow et al. ${ }^{28}$ These expansions can be written in the following condensed form:

$$
\begin{aligned}
& U_{i}(x, y)=\sum_{n=0}^{m} \sum_{j=0}^{n} \frac{\left[U_{n-j, j}\right]_{i} X^{n-j} Y^{j}}{(n-j) ! j !} \quad(i=1, \ldots, N) \\
& V_{i}(x, y)=\sum_{n=0}^{m} \sum_{j=0}^{n} \frac{\left[V_{n-j, j}\right]_{i} X^{n-j} Y^{j}}{(n-j) ! j !} \quad(i=1, \ldots, N)
\end{aligned}
$$

where $U_{i}(x, y)$ and $V_{i}(x, y)$ approximate the displacements $u$ and $v$, respectively, in the vicinity of the $i$ th nodal point. $\left[U_{n-j, j}\right]_{i}$ and $\left[V_{n-j, j}^{i}\right]_{i}$ are the coefficients of the Taylor series expansions and $m$ is its order. $N$ is the number of the nodal points. $X$ and $Y$ are the local co-ordinates with respect 


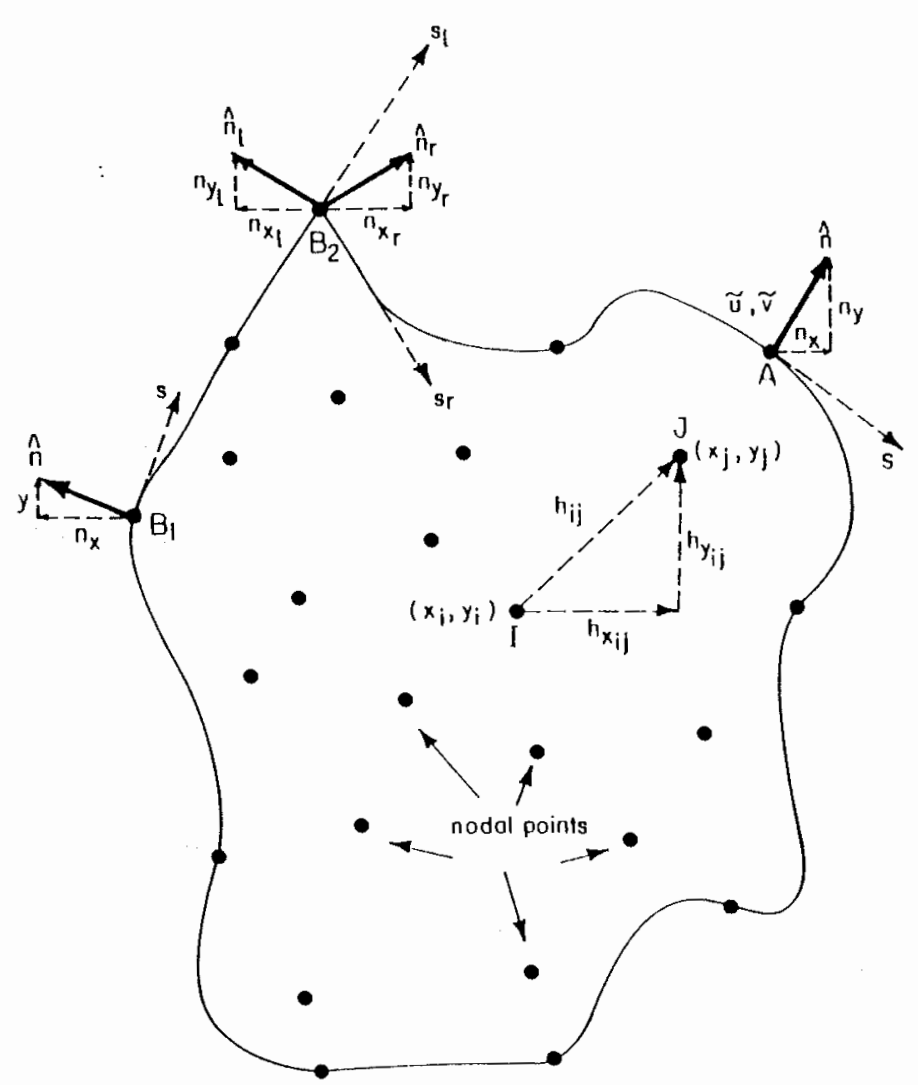

Figure 2. Schematic illustration of a general computation domain with definitions related to the nodal and neighbouring points

to the ith nodal point, i.e.

$$
X=x-x_{i} \text { and } Y=y-y_{i}
$$

where $x$ and $y$ are the global co-ordinates.

Note that the number of each of the coefficients $\left[U_{n-j, j}\right]_{i}$ and $\left[V_{n-j, j}\right]_{i}$ is $N_{\mathrm{c}}=\frac{1}{2}(m+1)(m+2)$.

The error of the above approximations is of the order $O\left(h^{m+1}\right)$ where, as mentioned earlier, $h$ is the grid size. For example, for a fourth-order Taylor series expansion (i.e. $m=4$ ) equation (6a) would explicitly read:

$$
\begin{aligned}
U_{i}(x, y)= & {\left[U_{0,0}\right]_{i}+\left[U_{1,0}\right]_{i} X+\left[U_{0,1}\right]_{i} Y } \\
& +\frac{1}{2}\left[U_{2,0}\right]_{i} X^{2}+\left[U_{1,1}\right]_{i} X Y+\frac{1}{2}\left[U_{0,2}\right]_{i} Y^{2} \\
& +\frac{1}{6}\left[U_{3,0}\right]_{i} X^{3}+\frac{1}{2}\left[U_{2,1}\right]_{i} X^{2} Y+\frac{1}{2}\left[U_{1,2}\right]_{i} X Y^{2}+\frac{1}{6}\left[U_{0,3}\right]_{i} Y^{3} \\
& +\frac{1}{24}\left[U_{4,0}\right]_{i} X^{4}+\frac{1}{6}\left[U_{3,1}\right]_{i} X^{3} Y+\frac{1}{4}\left[U_{2,2}\right]_{i} X^{2} Y^{2}+\frac{1}{6}\left[U_{1,3}\right]_{i} X Y^{3}+\frac{1}{24}\left[U_{0,4}\right]_{i} Y^{4} .
\end{aligned}
$$

It should also be noted here that the above-mentioned coefficients are indeed approximations of the unknown variables and their derivatives at the nodal points, i.e.

$\left[U_{0,0}\right]_{i}$ approximates $u$ if $\left(x_{i}, y_{i}\right)$, i.e. at $X=0$ and $Y=0$. 
$\left[V_{0,0}\right]_{i}$ approximates $v$ at $\left(x_{i}, y_{i}\right)$, i.e. at $X=0$ and $Y=0$. Similarly, $\left[U_{n-j, j}\right]_{i}$ approximates the derivative $\partial^{n} u / \partial x^{n-j} \partial y^{j}$ at $-\left(x_{i}, y_{i}\right)$, i.e. at $X=0$ and $Y=0$,
when $n \neq 0$, and

$\left[V_{n-j, j}\right]_{i}$ approximates the derivative $\partial^{n} v / \partial x^{n-j} \partial y^{j}$ at $\left(x_{i}, y_{i}\right)$, i.e. at $X=0$ and $Y=0$.

In the present illustration we adopt sixth-order Taylor series expansions (i.e. $m=6$ ) therefore, $N_{\mathrm{c}}=\frac{1}{2}(m+1)(m+2)=28$. Thus we have $28 U$-coefficients are $28 V$-coefficients for each one of the $N$ nodal points. Finding these 56 coefficients for each nodal point will provide the approxinated solution of the unknown variables. Note that the selection of a sixth-order Taylor series expansions ensures that the errors associated with them will be of the order $O\left(h^{7}\right)$. For small values of $h$ these are obviously very small errors.

Using ordinary finite difference methods implies that one has to use localized schemes of at least twenty-eight neighbouring values for each nodal point (see References 13, 23-26). By using our proposed DFD-method the number of the neighbouring values is reduced. This is illustrated in the following.

\section{Local governing equations}

The most common method for obtaining the values of the above-mentioned 56 unknown variables (i.e. the coefficient of the Taylor series expansions which approximate the solution, $\left[U_{n-j, j}\right]_{i}$ and $\left.\left[V_{n-j, j}\right]_{i}\right)$ is to:

(1) substitute the approximating Taylor series expansions [equations (6a) and (6b)] into the governing equations [equations (5a) and (5b)] for each nodal point while settiing the local co-ordinates, $X$ and $Y$, to be equal to zero, and

(2) require that the Taylor series expansions from the neighbouring points of a given nodal point will have the same value at that point.

As mentioned earlier, based on Jensen ${ }^{13}$ this requires the use of localized schemes (templates) having 28 neighbouring points. (Recall that sixth-order Taylor series expansions are used in this example.)

In order to obtain the 56 values of the unknown variables by using templates with less than 28 neighbouring points, we proposed to:

(1) successively differentiate the governing equations [equations (5a) and (5b)] and

(2) substitute the approximating Taylor series expansions [equations (6a) and (6b)] into both the governing equations and their differentiated equations, while setting the local coordinates, $X$ and $Y$, to be equal to zero.

The governing equations should be successively differentiated until the order of the derivatives equals the order of the Taylor series expansions. (e.g. six in the present example). Further differentiation will result in, after the substitution of the approximating Taylor series expansions, trivial equations.

Following the above description, the governing cquations [equations (5a) and (5b)] were successively differentiated to generate the following set of 28 equations.

differentiation by $\partial / \partial x$ yields

$$
\begin{aligned}
& c_{11} u_{x^{3}}+c_{33} u_{x y^{2}}+\left(c_{12}+c_{33}\right) v_{x^{2} y}=0 \\
& c_{33^{3}} v_{x^{3}}+c_{11} v_{x y^{2}}+\left(c_{12}+c_{33}\right) u_{x^{2} y}=0
\end{aligned}
$$


differentiation by $\partial / \partial y$ yields

$$
\begin{aligned}
& c_{11} u_{x^{2} y}+c_{33^{3}} u_{y^{3}}+\left(c_{12}+c_{33}\right) v_{x y^{2}}=0 \\
& c_{33} v_{x^{2} y}+c_{11} v_{y^{3}}+\left(c_{12}+c_{33}\right) u_{x y^{2}}=0
\end{aligned}
$$

differentiation by $\partial^{2} / \partial x^{2}$ yields

$$
\begin{aligned}
& c_{11} u_{x^{4}}+c_{33} u_{x^{2} y^{2}}+\left(c_{12}+c_{33}\right) v_{x^{3} y}=0 \\
& c_{33} v_{x^{4}}+c_{11} v_{x^{2} y^{2}}+\left(c_{12}+c_{33}\right) u_{x^{3} y}=0
\end{aligned}
$$

differentiation by $\partial^{2} / \partial x \partial y$ yields

$$
\begin{aligned}
& c_{11} u_{x^{3} y}+c_{33} u_{x y^{3}}+\left(c_{12}+c_{33}\right) v_{x^{2} y^{2}}=0 \\
& c_{33} v_{x^{3} y}+c_{11} v_{x y^{3}}+\left(c_{12}+c_{33}\right) u_{x^{2} y^{2}}=0
\end{aligned}
$$

differentiation by $\partial^{2} / \partial y^{2}$ yields

$$
\begin{aligned}
& c_{11} u_{x^{2} y^{2}}+c_{33} u_{y^{4}}+\left(c_{12}+c_{33}\right) v_{x y^{3}}=0 \\
& c_{33} v_{x^{2} y^{2}}+c_{11} v_{y^{4}}+\left(c_{12}+c_{33}\right) u_{x y^{3}}=0
\end{aligned}
$$

differentiation by $\partial^{3} / \partial x^{3}$ yields

$$
\begin{aligned}
& c_{11} u_{x^{5}}+c_{33} u_{x^{1} y^{2}}+\left(c_{12}+c_{33}\right) v_{x^{4} y}=0 \\
& c_{33} v_{x^{5}}+c_{11} v_{x^{3} y^{2}}+\left(c_{12}+c_{33}\right) u_{x^{4} y}=0
\end{aligned}
$$

differentiation by $\partial^{3} / \partial x^{2} \partial y$ yields

$$
\begin{aligned}
& c_{11} u_{x^{4} y}+c_{33} u_{x^{2} y^{3}}+\left(c_{12}+c_{33}\right) v_{x^{3} y^{2}}=0 \\
& c_{33} v_{x^{4} y}+c_{11} v_{x^{2} y^{3}}+\left(c_{12}+c_{33}\right) u_{x^{3} y^{2}}=0
\end{aligned}
$$

differentiation by $\partial^{3} / \partial x \partial y^{2}$ yields

$$
\begin{aligned}
& c_{11} u_{x^{3} y^{2}}+c_{33} u_{x y^{4}}+\left(c_{12}+c_{33}\right) v_{x^{2} y^{3}}=0 \\
& c_{33} v_{x^{3} y^{2}}+c_{11} v_{x y^{4}}+\left(c_{12}+c_{33}\right) u_{x^{2} y^{3}}=0
\end{aligned}
$$

differentiation by $\partial^{3} / \partial y^{3}$ yields

$$
\begin{aligned}
& c_{11} u_{x^{2} y^{3}}+c_{33} u_{y^{5}}+\left(c_{12}+c_{33}\right) v_{x y^{4}}=0 \\
& c_{33} v_{x^{2} y^{3}}+c_{11} v_{y^{5}}+\left(c_{12}+c_{33}\right) u_{x y^{4}}=0
\end{aligned}
$$

differentiation by $\partial^{4} / \partial x^{4}$ yields

$$
\begin{aligned}
& c_{11} u_{x^{6}}+c_{33} u_{x^{4} y^{2}}+\left(c_{12}+c_{33}\right) v_{x^{5} y}=0 \\
& c_{33} v_{x^{6}}+c_{11} v_{x^{4} y^{2}}+\left(c_{12}+c_{33}\right) u_{x^{5} y}=0
\end{aligned}
$$

differentiation by $\partial^{4} / \partial x^{3} \partial y$ yields

$$
\begin{aligned}
& c_{11} u_{x^{3} y}+c_{33} u_{x^{3} y^{3}}+\left(c_{12}+c_{33}\right) v_{x^{4} y^{2}}=0 \\
& c_{33} v_{x^{3} y}+c_{11} v_{x^{3} y^{3}}+\left(c_{12}+c_{33}\right) u_{x^{4} y^{2}}=0
\end{aligned}
$$

differentiation by $\partial^{4} / \partial x^{2} \partial y^{2}$ yields

$$
\begin{aligned}
& c_{11} u_{x^{4} y^{2}}+c_{33} u_{x^{2} y^{4}}+\left(c_{12}+c_{33}\right) v_{x^{3} y^{3}}=0 \\
& c_{33} v_{x^{4} y^{2}}+c_{11} v_{x^{2} y^{4}}+\left(c_{12}+c_{33}\right) u_{x^{3} y^{3}}=0
\end{aligned}
$$


differentiation by $\partial^{4} / \partial x \partial y^{3}$ yields

$$
\begin{aligned}
& c_{11} u_{x^{3} y^{3}}+c_{33} u_{x y^{5}}+\left(c_{12}+c_{33}\right) v_{x^{2} y^{4}}=0 \\
& c_{33} v_{x^{3} y^{3}}+c_{11} v_{x y^{5}}+\left(c_{12}+c_{33}\right) u_{x^{2} y^{4}}=0
\end{aligned}
$$

differentiation by $\partial^{4} / \partial y^{4}$ yields

$$
\begin{aligned}
& c_{11} u_{x^{2} y^{4}}+c_{33} u_{y^{6}}+\left(c_{12}+c_{33}\right) v_{x y}=0 \\
& c_{33} v_{x^{2} y^{4}}+c_{11} v_{y^{6}}+\left(c_{12}+c_{33}\right) u_{x y},-0
\end{aligned}
$$

It can be seen that the number of the equations which results from the performance of the above illustrated differentiation procedure together with the appropriate governing equations is:

$$
N_{\mathrm{e}}=\frac{[(m-k)+1][(m-k)+2]}{2}
$$

for each one of the governing equations [equations (5a) and (5b)]. Here $m$ is the order of the Taylor series expansions and $k$ is the order of the lowest derivative of the unknown variables in the governing equations. For example, it is evident from equations (5a) and (5b) that in the present example $k=2$, in addition since we chose $m=6$, the above expression implies that $N_{\mathrm{e}}=15$ as is the case in equations (5) and (7). It is interesting to note that development of the Modified Equivalent Partial Differential Equations (MEPDE) involved successive differentiation of the kind used to develop equations (7a)-(7n).

Recall that as shown in the foregoing discussion $\left[U_{n-j, j}\right]_{i}$ and $\left[V_{n-j, j}\right]_{i}$ approximate the values of $u$ and $v$, respectively, at $\left(x_{i}, y_{i}\right)$ i.e. at $X=0$ and $Y=0$. Therefore, if the approximations given by equations [(6a) and (6b)] are substituted into the governing equations [(5a) and (5b)] and their differentiated equations $[(7 \mathrm{a})-(7 \mathrm{n})]$ and $X$ and $Y$ are set to zero for the $i$ th nodal point (i.e. the Taylor series approximations satisfy the differential equations at the nodal points) one obtains a set of algebraic equations identical to equations (5) and (7) in which the coefficients $\left[U_{n-j, j}\right]_{i}$ and $\left[V_{n-j, j}\right]_{i}$ replace, the derivatives $U_{x^{n-j_{y}} j}$ and $V_{x^{n-j_{y} j}}$, respectively. These set of equations will be referred to in the following as the local governing equations.

For example, the governing equations (5a) and $(5 b)$ will become

$$
\begin{aligned}
& c_{11}\left[U_{2,0}\right]_{i}+c_{33}\left[U_{0,2}\right]_{i}+\left(c_{12}+c_{33}\right)\left[V_{1,1}\right]_{i}=0 \\
& c_{33}\left[V_{2,0}\right]_{i}+c_{11}\left[V_{0,2}\right]_{i}+\left(c_{12}+c_{33}\right)\left[U_{1,1}\right]_{i}=0
\end{aligned}
$$

Similarly, the differentiated equations given by $(7 \mathrm{n})$ will read

$$
\begin{aligned}
& c_{11}\left[U_{2,4}\right]_{i}+c_{33}\left[U_{0,6}\right]_{i}+\left(c_{12}+c_{33}\right)\left[V_{1,5}\right]_{i}=0 \\
& c_{33}\left[V_{2,4}\right]_{i}+c_{11}\left[V_{0,6}\right]_{i}+\left(c_{12}+c_{33}\right)\left[U_{1,5}\right]_{i}=0
\end{aligned}
$$

At this stage one has $N_{\mathrm{c}}$ coefficients and $N_{\mathrm{e}}$ local governing equations [e.g. 28 coefficients and 15 equations for each variable ( $u$ or $v$ ) in the present example]. Consequently, in order to have a solvable set of equations one must generate $\left(N_{\mathrm{c}}-N_{\mathrm{e}}\right)$ additional equations (e.g. 13 equations in the present example) for each variable.

The procedure for generating the additional 13 equations for each unknown is to use 14-point templates (i.e. one central nodal point and 13 neighbouring points). This approach is common when finite difference methods are applied to an arbitrary mesh. For example see References 13, 23 and 26 . For the reader's clarity this is shown in the following section. 
The procedure for generating these additional equations is illustrated in Figure 2. At the first step $\left(N_{\mathrm{c}}-N_{\mathrm{e}}\right)$ neighbouring points indicated by $J$, are selected for each central point $I$. A way of selection ensuring no singularity or ill-conditioning is described subsequently. Let us denote the horizontal and vertical distances from the central point, $l$, to each neighbouring point, $J$, by $h_{x_{i j}}$ and $h_{y_{i}}$, respectively. Since the values of $u$ and $v$ at point $J$, as calculated by the Taylor series expansions from points $I$ or $J$, should be equal (within the error associated with the Taylor series expansions), one can write, in general, for each one of the neighbouring points

$$
\begin{aligned}
& U_{i}\left(x_{j}, y_{j}\right)-U_{j}\left(x_{j}, y_{j}\right)=0 \pm \varepsilon \\
& V_{i}\left(x_{j}, y_{j}\right)-V_{j}\left(x_{j}, y_{j}\right)=0 \pm \varepsilon
\end{aligned}
$$

where $U_{i}\left(x_{j}, y_{j}\right)$ and $V_{i}\left(x_{j}, y_{j}\right)$ can be simply obtained from equations (6a) and $(6 \mathrm{~b})$, respectively, by inserting into them $X=h_{x_{i j}}$ and $Y=h_{y_{i j}}$. Similarly, $U_{j}\left(x_{j}, y_{j}\right)==\left[U_{0.0}\right]_{j}$ and $V_{j}\left(x_{j}, y_{j}\right)=\left[V_{0,0}\right]_{j}$ since for the neighbouring point $j, X=0$ and $Y=0$. These will result in the following set of 13 fitting equations for each variable.

$$
\begin{gathered}
\sum_{n=0}^{m} \sum_{k=0}^{n} \frac{\left[U_{n-k, k}\right]_{i} h_{x}^{n-k} h_{y}^{k}}{(n-k) ! k !}-\left[U_{0,0}\right]_{j}=0 \pm \varepsilon \\
\sum_{n=0}^{m} \sum_{k=0}^{n} \frac{\left[V_{n-k, k}\right]_{i} h_{x}^{n-k} h_{y}^{k}}{(n-k) ! k !}-\left[V_{0.0}\right]_{j}=0 \pm \varepsilon
\end{gathered}
$$

where, for simplicity, $h_{x i}$ and $h_{y, j}$, as denoted in Figure 2, are replaced by $h_{x}$ and $h_{y}$, respectively. The error, $\varepsilon$, is of the order $O\left(h_{i j}^{m+1}\right)$ where $h_{i j}^{2}=h_{x i j}^{2}+h_{y_{i j}}^{2}$.

The foregoing procedure of generating the fitting equations [(9a) and (9b)] should be applied to any internal nodal point (e.g. point $I$ in Figure 2). For nodal points which are located on the boundary, a different procedure which eventually results in a further reduction in the total number of the required neighbouring points is suggested in the following.

The use of the boundary conditions

Primary (displacement) boundary conditions. Let us first consider a nodal point located on the boundary whose boundary conditions are given by equation (2). Point $A$ in Figure 2 is such a nodal point. Since the displacements $u$ and $v$ (which are the unknown variables) are given at this point as $\hat{u}$ and $\hat{v}$, respectively, we simply write

$$
\begin{aligned}
& {\left[U_{0,0}\right]_{A}=\tilde{u}} \\
& {\left[V_{0,0}\right]_{A}=\tilde{v}}
\end{aligned}
$$

Hence, if point $A$ is selected to be the neighbouring point to an internal point, $\left[U_{0.0}\right]_{j}$ and $\left[V_{0,0}\right]_{j}$ in equations $(9 \mathrm{a})$ and $(9, \mathrm{~b})$ are, respectively, replaced by $\left[U_{0,0}\right]_{A}$ and $\left[V_{0.0}\right]_{A}$.

Natural (traction) boundary conditions. The case is different when the boundary conditions of a nodal point which is located on the boundary are given by equation (3). In this case we distinguish between a point at a regular boundary and a point at a corner (e.g. points $B_{1}$ and $B_{2}$, respectively, in Figure 2): A similar distinction was noted by Dow et al. ${ }^{26}$ 
Regular boundary

For a nodal point such as $B_{1}$ we have the following traction boundary conditions:

$$
\begin{aligned}
& \left(c_{11} u_{x}+c_{12} v_{y}\right) n_{x}+c_{33}\left(u_{y}+v_{x}\right) n_{y}=\tilde{t}_{x} \\
& c_{33}\left(u_{y}+v_{x}\right) n_{x}+\left(c_{12} u_{x}+c_{11} v_{y}\right) n_{y}=\tilde{t}_{y}
\end{aligned}
$$

Since the distributions of the boundary conditions along the boundary are also given and since the boundary conditions are differential equations having an order $k_{1}$, where $k_{1}<k$ [here $k$ is the order of the governing equations e.g. $k=2$ in equations (5a) and (5b) and $k_{1}=1$ in equations $(10 \mathrm{a})$ and $(10 \mathrm{~b})]$, we can successively differentiate the boundary conditions $\left(m-k_{1}\right)$ times along the boundary. This yields the following set of $\left(m-k_{1}\right)$ equations for each one of the boundary conditions given by equations (10a) and (10b). Here, $s$ is the tangential direction of the boundary at the point $B_{1}$ as shown in Figure 2.

$$
\begin{aligned}
& \frac{\partial^{n}}{\partial s^{n}}\left[\left(c_{11} u_{x}+c_{12} v_{y}\right) n_{x}+c_{33}\left(u_{y}+v_{x}\right) n_{y}\right]=\frac{\partial^{n}}{\partial s^{n}}\left(\tilde{t}_{x}\right), \quad n=1,2, \ldots,\left(m-k_{1}\right) \\
& \frac{\partial^{n}}{\partial s^{n}}\left[c_{33}\left(u_{y}+v_{x}\right) n_{x}+\left(c_{12} u_{x}+c_{11} v_{y}\right) n_{y}\right]=\frac{\partial^{n}}{\partial s^{n}}\left(\tilde{t}_{y}\right)
\end{aligned}
$$

Inserting the approximations given by equations (6a) and (6b) into equations (10) and (11) and setting $X=0$ and $Y=0$ results in a set of $N_{\mathrm{b}}=\left[\left(m-k_{1}\right)+1\right]$ equations for each of the unknown variables. These equations will be referred to in the following as the local boundary condition equations. Thus, for a nodal point on the boundary such as $B_{1}$ (see Figure 2), one has $N_{\mathrm{e}}$ local governing equations and $N_{\mathrm{b}}$ local boundary condition equations. Consequently $\left[N_{\mathrm{c}}-\left(N_{\mathrm{e}}+N_{\mathrm{b}}\right)\right]$ neighbouring points should be selected in order to generate the appropriate fitting equations [e.g. $N_{\mathrm{c}}=28, N_{\mathrm{e}}=15, N_{\mathrm{b}}=6$ and hence seven neighbouring points are required in the present example]. This results in local schemes (templates) of eight points.

The foregoing description of generating the local boundary condition equations is illustrated, for the reader's convenience, in the following example.

Let us consider a straight oblique boundary as the one shown in Figure 3. It could be shown that for any differentiable and continuous function $f$ one can write

$$
\frac{\mathrm{d} f}{\mathrm{~d} s}=\frac{\partial f}{\partial x} \frac{\partial x}{\partial s}+\frac{\partial f}{\partial y} \frac{\partial y}{\partial s}
$$

It is clear from Figure 3 that

$$
\frac{\partial x}{\partial s}=-\sin \theta=-n_{y} \quad \text { and } \quad \frac{\partial y}{\partial s}=\cos \theta=n_{x}
$$

thus

$$
\frac{\mathrm{d} f}{\mathrm{~d} s}=-\frac{\mathrm{d} f}{\mathrm{~d} x} n_{y}+\frac{\mathrm{d} f}{\mathrm{~d} y} n_{x}
$$

Let us assume that the boundary conditions are given by

$$
\begin{aligned}
& \tilde{t}_{x}=3 x+\sin y+y^{2}+2 x^{2} y \\
& \tilde{t}_{y}=5 x^{2}+\cos x+4 x^{2} y^{2}+6 y+12
\end{aligned}
$$




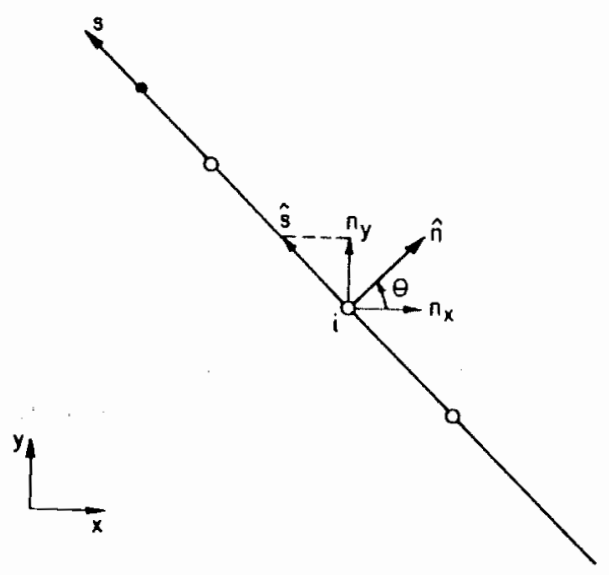

Figure 3. Definition of the various parameters associated with a straight oblique boundary

Equations (11a) and (11b) imply for $n=1$ :

Similarly

$$
\begin{aligned}
& -\frac{\partial}{\partial x}\left[\left(c_{11} u_{x}+c_{12} v_{y}\right) n_{x}+c_{33}\left(u_{y}+v_{x}\right) n_{y}\right] n_{y} \\
& +\frac{\partial}{\partial y}\left[\left(c_{11} u_{x}+c_{12} v_{y}\right) n_{x}+c_{33}\left(u_{y}+v_{x}\right) n_{y}\right] n_{x} \\
& =-\frac{\partial}{\partial x}\left(\tilde{t}_{x}\right) n_{y}+\frac{\partial}{\partial y}\left(\tilde{t}_{x}\right) n_{x}
\end{aligned}
$$

$$
\begin{aligned}
& -\frac{\partial}{\partial x}\left[c_{33}\left(u_{y}+v_{x}\right) n_{x}+\left(c_{12} u_{x}+c_{11} v_{y}\right) n_{y}\right] n_{y} \\
& +\frac{\partial}{\partial y}\left[c_{33}\left(u_{y}+v_{x}\right) n_{x}+\left(c_{12} u_{x}+c_{11} v_{y}\right) n_{y}\right] n_{x} \\
& =-\frac{\partial}{\partial x}\left(\tilde{I}_{y}\right) n_{y}+\frac{\partial}{\partial y}\left(\tilde{t}_{y}\right) n_{x}
\end{aligned}
$$

Inserting the boundary conditions given in the above example into these two expressions results in

$$
\begin{gathered}
-c_{11} n_{x} n_{y} u_{x x}+\left(c_{11} n_{x}^{2}-c_{33} n_{y}^{2}\right) u_{x y}+c_{33} n_{x} n_{y} u_{y y} \\
-c_{33} n_{y}^{2} v_{x x}+\left(c_{33}-c_{12}\right) n_{x} n_{y} v_{x y}+c_{12} n_{x}^{2} v_{y y} \\
=-(3+4 x y) n_{y}+\left(\cos y+2 y+2 x^{2}\right) n_{x}
\end{gathered}
$$

and

$$
\begin{aligned}
& -c_{12} n_{y}^{2} u_{x x}+\left(c_{12}-c_{33}\right) n_{x} n_{y} u_{x y}+c_{33} n_{x}^{2} u_{y y} \\
& -c_{33} n_{x} n_{y} v_{x x}+\left(c_{33} n_{x}^{2}-c_{11} n_{y}^{2}\right) v_{x y}+c_{11} n_{x} n_{y} v_{y y} \\
& =-\left(10 x-\sin x+8 x y^{2}\right) n_{y}+\left(8 x^{2} y+6\right) n_{x}
\end{aligned}
$$


Inserting the approximating Taylor series expansion given by equations (6a) and (6b) while setting $X=0$ and $Y=0$ (i.e. $x=x_{i}$ and $y=y_{i}$ since as mentioned earlier $X=x-x_{i}$ and $\left.Y=y-y_{i}\right)$ yields

$$
\begin{aligned}
& -c_{11} n_{x} n_{y}\left[U_{2,0}\right]_{i}+\left(c_{11} n_{x}^{2}-c_{33} n_{y}^{2}\right)\left[U_{1,1}\right]_{i}+c_{33} n_{x} n_{y}\left[U_{0.2}\right]_{i} \\
& -c_{33} n_{y}^{2}\left[V_{2,0}\right]_{i}+\left(c_{33}-c_{12}\right) n_{x} n_{y}\left[V_{1,1}\right]_{i}+c_{12} n_{x}^{2}\left[V_{0,2}\right]_{i} \\
& =-\left(3+4 x_{i} y_{i}\right) n_{y}+\left(\cos y_{i}+2 y_{i}+2 x_{i}^{2}\right) n_{x}
\end{aligned}
$$

and

$$
\begin{aligned}
& \left.-c_{12} n_{y}^{2}\left[U_{2,0}\right]_{i}+\left(c_{12}-c_{33}\right) n_{x} n_{y}\right)\left[U_{1,1}\right]_{i}+c_{33} n_{x}^{2}\left[U_{0.2}\right]_{i} \\
& -c_{33} n_{x} n_{y}\left[V_{2,0}\right]_{i}+\left(c_{33} n_{x}^{2}-c_{11} n_{y}^{2}\right)\left[V_{1,1}\right]_{i}+c_{11} n_{x} n_{y}\left[V_{0,2}\right]_{i} \\
& =-\left(10 x_{i}-\sin x_{i}+8 x_{i} y_{i}^{2}\right) n_{y}+\left(8 x_{i}^{2} y_{i}+6\right) n_{x}
\end{aligned}
$$

Recall that in order to generate the full set of the local boundary condition equations, equations (I Ia) and (11b) should be executed also for $n=2, \ldots, 5$, since in the above example $m=6$ and $k_{1}=1$.

\section{Corner}

For a corner nodal point such as $B_{2}$ we have two sets of traction boundary conditions. One set to its left, 1 , and the other to its right, $r$. Each set is identical to equations (10a) and (10b) with its appropriate $n_{x}$ and $n_{y}$ (recall that $B_{2}$ is located at a corner).

Careful inspection of equations (10a) and (10b) indicates that they contain the following three unknowns: the normal stresses $\sigma_{x x}=c_{11} u_{x}+c_{12} v_{y}$ and $\sigma_{y y}=c_{12} u_{x}+c_{11} v_{y}$ and the shear stress $\tau_{x y}=c_{33}\left(u_{y}+v_{x}\right)$. Thus, using the two sets (left and right) for a corner nodal point will result in four equations with only three unknowns. In fact these four equations are linearly dependent. Furthermore, it can be shown that any three of them are linearly independent. Therefore, one of them should be arbitrarily dropped. Thus differentiating each set with respect to $s_{1}$ or $s_{\mathrm{r}}$, respectively, (see Figure 2) results in $\left(4 N_{\mathrm{b}}-1\right)$ local boundary condition equations $\left[4 N_{\mathrm{b}}\right.$ as a result of differentiating equations (10a) and (10b) to the left and to the right of the corner nodal point and -1 due to the arbitrary drop of redundant boundary conditions]. Hence, for a corner nodal point such as $B_{2}$, one has $2 N_{\mathrm{e}}$ local governing equations and $\left(4 N_{\mathrm{b}}-1\right)$ local boundary condition equations. Consequently, $\left[2 N_{\mathrm{c}}-\left(2 N_{\mathrm{e}}+4 N_{\mathrm{b}}-1\right)\right]$ fitting equations should be added [e.g. $N_{\mathrm{c}}=28, N_{\mathrm{e}}=15, N_{\mathrm{b}}=6$ and only three fitting equations are required in the present example]. This is done by selecting two neighbouring points and fitting to one of them only one of the two equations given by equations $(9 \mathrm{a})$ and $(9 \mathrm{~b})$. This nodal point acts in fact like a hal $\Gamma$ neighbouring point.

In summary, for the illustrated case, i.e. the equilibrium equations in terms of displacements in plane elasticity, as given by (5a) and ( $5 \mathrm{~b}$ ), and the appropriate boundary conditions, there is a need for 13 neighbouring points (14-point templates) for each internal nodal point (such as point $I$ in Figure 2), 7 neighbouring points (8-point templates) for a nodal point along a regular boundary (such as point $B_{1}$ in Figure 2 ) and $1 \frac{1}{2}$ neighbouring points ( $2 \frac{1}{2}$-point templates) for a corner nodal point (such as point $B_{2}$ in Figure 2). This is summarized for the reader's convenience in Table 1 .

It should be emphasized that for nodal points located on a boundary with natural (traction) boundary conditions, the proposed method requires to fit the approximating Taylor series expansions (6a) and (6b) to the governing equations (5a) and (5b), their differentiated equations (7a) and (7n), the boundary conditions (10a) and (10b) and their differentiated equations (11a) and 


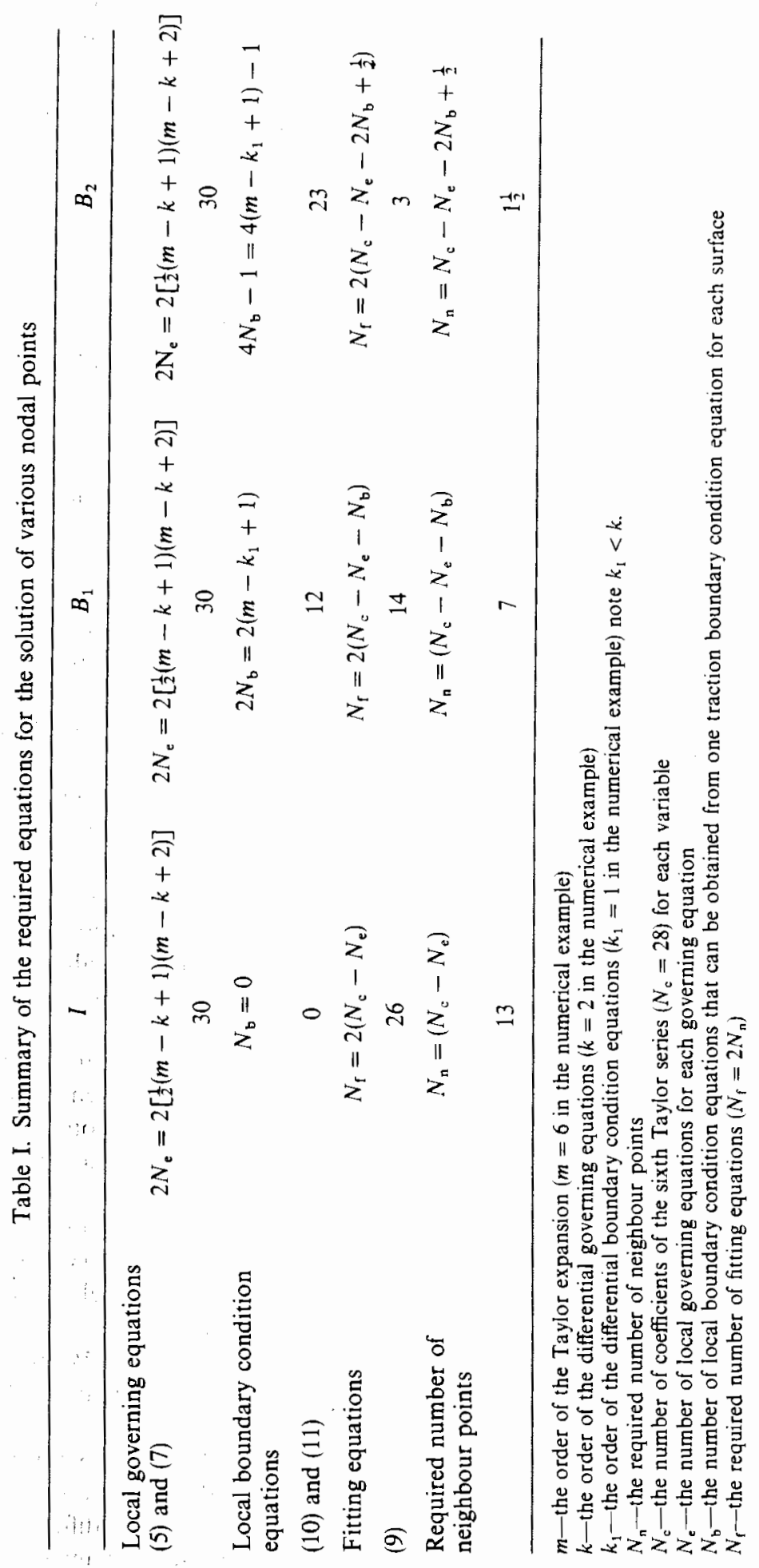


(11b). In addition, there is a requirement to fit the various Taylor series expansions which are developed from its neighbouring points and the one developed from it (i.e., the fitting equations). The above is accomplished without the need to use fictitious nodal points. Note that the use of fictitious nodal points increases the order of the error in addition to the redundant computations associated with them. This was clearly described by Noye and Arnold (1990). The abovementioned requirements together with the fact that the proposed method does not require the use of fictitious nodal points results in an improvement in the boundary value approximation with respect to ordinary finite difference methods.

\section{SOLUTION METHOD}

The foregoing described procedure enables one to generate 56 equations for each one of the $N$ nodal points. Thus, the $56 \mathrm{~N}$ unknown coefficients $\left(2 N_{\mathrm{c}}\right)$ can in principal be solved, e.g. by inverting a $56 \mathrm{~N} \times 56 \mathrm{~N}$ matrix. For any reasonable value of $N$ this would be a very inefficient and ineffective way. Due to this complexity it is common to define a local matrix for each nodal point $(56 \times 56$ in the present illustration) and then to further reduce it.

Kochavi et al. ${ }^{32}$ gave a procedure by which the solution can be reduced to the inversion of a $54 \times 54$ local matrix for each one of the $N$ nodal points, and eventually obtain $2 N$ equations with $2 N$ unknowns, i.e. $\left[U_{0,0}\right]_{i}$ and $\left[V_{0,0}\right]_{i}$ for each one of the $N$ nodal points. Similar approaches can be seen in References 23 and 26.

In the following we present and formulate the method by which the solution is further reduced to the inversion of a $26 \times 26$ matrix for a type $I$ nodal point, $14 \times 14$ matrix for a type $B_{1}$ nodal point and $3 \times 3$ matrix for a type $B_{2}$ nodal point. (Recall that the nodal types are defined in Figure 2.) Note that the matrix size is identical to the number of the fitting equations which are required. This formulation should not be regarded as a conditional part of our proposed method. In the authors' view it reduces the computation volume. However, in principle, any other way of solving the equations can be adopted.

\section{Proposed solution method}

The set of equations at each nodal point which is composed of local governing equations, local boundary condition equations and fitting equations can in general be written as

$$
[K]\{A\}=\{F\}
$$

where $[K]$ is a matrix of order $56 \times\left(56+N_{\mathrm{f}}\right)$; here $N_{\mathrm{f}}$ is the number of the fitting equations (for details, see Table 1$),\{A\}$ is a column vector of dimension $\left(56+N_{i}\right)$ containing the 56 unknowns of the nodal point and the $N_{f}$ unknowns of the neighbouring points, i.e. $\left[U_{0,0}\right]_{j}$ and $\left[V_{0,0}\right]_{j}$ for $j=1, \ldots, N_{\mathrm{n}}$, where $N_{\mathrm{n}}$ the number of the neighbouring points is equal to $\frac{1}{2} N_{\mathrm{f}}$.

We now divide the vector $\{A\}$ into three subvectors: $\left\{A_{1}\right\}$ contains all the $\left[U_{0,0}\right]$ and $\left[V_{0,0}\right]$ values (both of the $i$ th nodal points and its $N_{\mathrm{n}}$ neighbouring points); hence its dimension is $2\left(N_{n}+1\right)$ or alternatively $\left(N_{\mathrm{f}}+2\right)$. The rest of the unknowns (i.e. $\left[U_{p, q}\right]_{i}$ and $\left[V_{p, q}\right]_{i}$ for which $p+q \neq 0)$ are divided in the following way: the first $\frac{1}{2} N_{\mathrm{r}}$ unknowns of each of two Taylor series expansions given by equations (6a) and (6b) form the vector $\left\{A_{2}\right\}$ which therefore has a dimension $N_{\mathrm{f}}$, and the rest of them form the vector $\left\{A_{3}\right\}$ whose dimension is $\left(54-N_{f}\right)$. 
As a result of this division, equation (12) breaks into the following form:

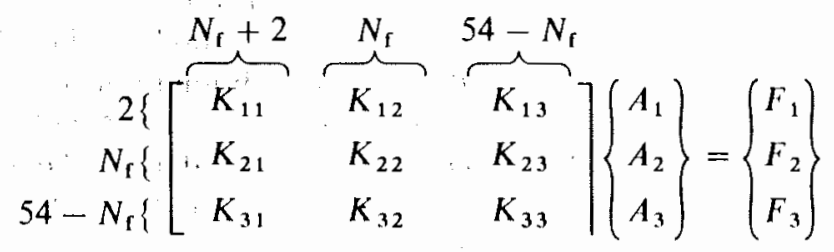

Alternatively, this can be split into the following three matrix equations:

$$
\left[K_{11}\right]\left\{A_{1}\right\}+\left[K_{12}\right]\left\{A_{2}\right\}+\left[K_{13}\right]\left\{A_{3}\right\}=\left\{F_{1}\right\}
$$

which are the two approximations of the governing equations (5a) and (5b),

$$
\left[K_{21}\right]\left\{A_{1}\right\}+\left[K_{22}\right]\left\{A_{2}\right\}+\left[K_{23}\right]\left\{A_{3}\right\}=\left\{F_{2}\right\}
$$

which are the fitting equations $(9 \mathrm{a})$ and $(9 \mathrm{~b})$, and

$$
\left[K_{31}\right]\left\{A_{1}\right\}+\left[K_{32}\right]\left\{A_{2}\right\}+\left[K_{33}\right]\left\{A_{3}\right\}=\left\{F_{3}\right\}
$$

which are the remaining local governing, equations $(7 a)$ and $(7 b)$, and the local boundary condition, equations (10a), (10b), (11a) and (11b), when the nodal point is on the boundary.

Prior to proceeding with the solution of equations (14a) and (14b) it should be noted that both $\left[K_{13}\right]$ and $\left[K_{31}\right]$ are in general zero matrices. This is due to the fact that based on the procedure by which $\left[K_{31}\right]$ is generated, it contains only derivatives of the unknowns. Equation (14a) which contains only low-order derivatives, i.e. those appearing in the governing equations, implies that in general $\left[K_{13}\right]=0$ (e.gi second order derivatives in the present example). In addition, it could be seen from the fitting equations $(9 \mathrm{a})$ and $(9 \mathrm{~b})$ that the vector $\left\{F_{2}\right\}=0$.

Inserting these facts into equations (14a) and (14c) and solving the entire set for $\left\{A_{1}\right\}$ finally results in:

$$
\begin{aligned}
& {\left[\left[K_{11}\right]-\left[K_{12}\right]\left[\left[K_{22}\right]-\left[K_{23}\right]\left[K_{33}\right]^{-1}\left[K_{32}\right]\right]^{-1}\left[K_{21}\right]\right]\left\{A_{1}\right\}} \\
& \quad=\left\{F_{1}\right\}+\left[K_{12}\right]\left[\left[K_{22}\right]-\left[K_{23}\right]\left[K_{33}\right]^{-1}\left[K_{32}\right]\right]^{-1}\left[K_{23}\right]\left[K_{33}\right]^{-1}\left\{F_{3\}}\right\}
\end{aligned}
$$

The matrix equation (15) which applies to each one of the $N$ nodal points consists of two equations which, as mentioned, earlier, contain only $\left[U_{0,0}\right]$ and $\left[V_{0.0}\right]$ unknowns. Thus, at this stage we have $2 N$ equations with $2 N$ unknowns. These equations can be written in the following matrix form:

$$
[G][\phi]=[S]
$$

where $[\phi]$ is the unknown vector (it contains $\left[U_{0,0}\right]$ and $\left[V_{0,0}\right]$ ) and $[G]$ and $[S]$ are simply obtained from equation (15). These equations can be solved by standard solution methods (e.g. iterative or direct methods).

Once the values of $\left[U_{0,0}\right]_{i}$ and $\left[V_{0,0}\right]_{i}$ are obtained (i.e. $\left\{A_{1}\right\}$ is known) for alt the $N$ nodal points, the appropriate approximations of the derivatives $\left[U_{p, q}\right]_{i}$ and $\left[V_{p, q}\right]_{i}$ where $p+q \neq 0$ could be obtained from equations (14a), (14b) and (14c) by solving for $\left\{A_{2}\right\}$ and $\left\{A_{3}\right\}$.

It should be pointed out here that the coefficient matrices $\left[K_{11}\right],\left[K_{12}\right]$ and $\left[K_{13}\right]$ which arise from the approximations of the governing equations are the same for all the nodal points; the coefficient matrices $\left[K_{31}\right],\left[K_{32}\right]$ and $\left[K_{33}\right]$ are the same for all the $I$-type, points (see Figure 2); and only the coefficient matrices $\left[K_{21}\right],\left[K_{22}\right]$ and $\left[K_{23}\right]$ which arise from the fitting equations differ from point to point as they depend on the geometry of the investigated problem, or the 
relative location of the neighbouring points. This minor complexity can be simplified by arranging the nodal points in such a way that identical geometrical patterns of neighbouring points (templates) are generated. Therefore, the coefficient matrices $\left[K_{11}\right],\left[K_{12}\right]$ and $\left[K_{13}\right]$ should be calculated only once, the coefficient matrices $\left[K_{31}\right],\left[K_{32}\right]$ and $\left[K_{33}\right]$ should be calculated only once for all the $I$-type nodal points. Note that this implies that $\left[K_{33}\right]$ which appears in equation (15) should be inverted at most three times. The coeflicient matrices $\left[K_{21}\right]$, $\left[K_{22}\right]$ and $\left[K_{23}\right]$ should be calculated once for each geometrical pattern (template). The above discussion implies that $\left[\left[K_{22}\right]-\left[K_{23}\right]\left[K_{33}\right]^{-1}\left[K_{32}\right]\right]$ which also appears in equation (15) should be calculated and inverted only once for each template.

Note, that as mentioned earlier, the size of this coefficient matrix is $26 \times 26$ for an $I$-type nodal point, $14 \times 14$ for a $B_{1}$-type nodal point and $3 \times 3$ for a $B_{2}$-type nodal point. It is the only matrix which should be inverted for each one of the different geometrical patterns. As a consequence, the coefficient matrices in equation (15) are identical for each of the different geometrical patterns. The only change between the various geometrical patterns is in the vectors $\left\{A_{i}\right\}$ and $\left\{F_{i}\right\}$.

\section{SINGULARITIES OR ILL-CONDITIONS IN MATRICES}

In general, two problems related to singularities or ill-conditions in matrices exist in numerical methods. The first problem can appear in the local matrix while the second in the global one. In the following they will be discussed separately.

\section{Local matrix}

The local matrix in the present solution method is the one marked by $[K]$ in equation (12). As seen in equation (15) the proposed solution method involved the inversion of

$$
\left[\left[K_{22}\right]-\left[K_{23}\right]\left[K_{33}\right]^{-1}\left[K_{32}\right]\right]^{-1}
$$

Therefore one must assure that the above matrix is not singular or ill-conditioned. (Recall that as mentioned earlier $\left[K_{33}\right]$, which does not depend on the local schemes, cannot be singular.)

Perrone and $\mathrm{KaO}^{23}$ treated the singularity and ill-conditioning of their local matrix (which was slightly different from the present one) and suggested a technique (criterion) to avoid the singularity. Liszka and Orkisz ${ }^{24}$ proposed a different technique which they claimed was simpler and quicker in addition to being more accurate.

Unfortunately, Perrone and Kao's ${ }^{23}$ criterion is limited to maximum eight neighbouring points (i.e. templates of nine nodal points), and Liszka and Orkisz's ${ }^{24}$ criterion does not always guarantee non-singularity.

Perrone and Kao's ${ }^{23}$ criterion is illustrated in Figure 4. The area around the central nodal point, $C$, is divided into eight different zones each of which is a pie-shaped segment with a central angle of $45^{\circ}$. Segments I and III are bisected by the $X$-axis and segments II and IV are bisected by the $Y$-axis. Segments V-VIII are bisected by $45^{\circ}$ lines through the nodal point, $C$. For an $\mathrm{N}$-point template (in which $5 \leqslant N \leqslant 9$ ) the criterion suggests to locate the first four neighbouring points in segments I-IV and the rest of them in the other segments (at most one point per segment).

Since our method allows templates having more than nine points we propose to distribute the neighbouring points in the following way. They should occupy all the eight segments and should be located as far as possible from each other while being close enough to the central nodal point. 


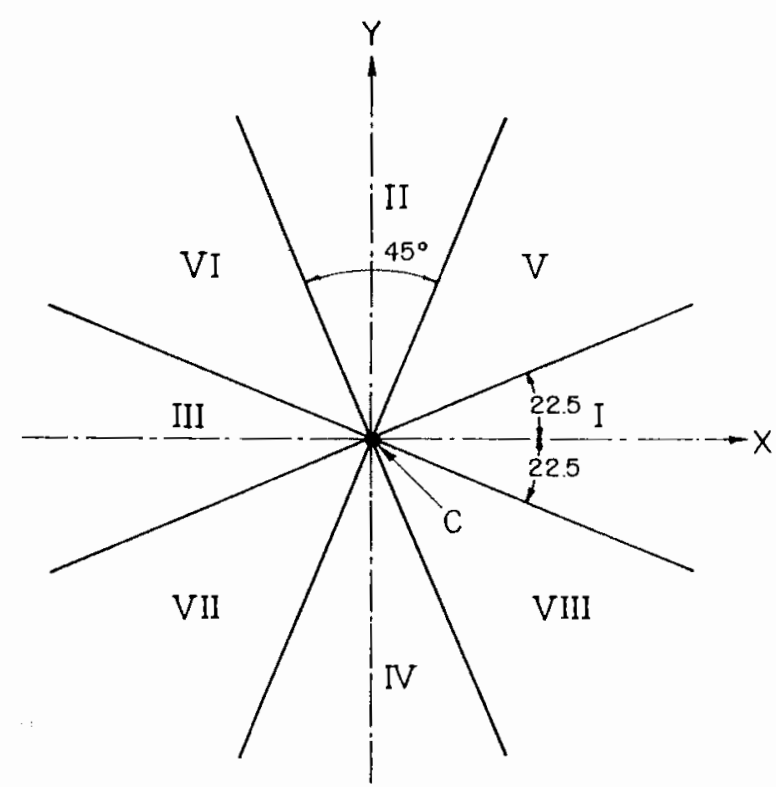

Figure 4. Perrone and $\mathrm{Kao}^{\prime} \mathrm{s}^{23}$ eight-segment configuration for selecting the local schemes (templates)

\section{Global matrix}

The global matrix, i.e. [G] in equation (16), cannot be singular. It can, however, be illconditioned. The ill-conditioning can be weakened by ensuring that

$$
\left|g_{i i}\right| \geqslant\left|g_{i j}\right|
$$

for all the rows $-i$. $\left(g_{i i}\right.$ and $g_{i j}$ are the terms of $[G]$ ). Note that the above requirement is not necessary. However, the more rows fulfilling it, the further away is the matrix from ill-conditioning. By redistributing the neighbouring points, problematic rows which do not fulfill the above-mentioned requirement can be improved.

\section{EXAMPLES, VERIFICATION AND DISCUSSION}

In the following, two examples for which the analytical solution is known will be solved using the proposed method.

The first example is shown in Figure 5. A thin rectangular plate, having a dimension of $l \times b$, is fixed at its left edge. The boundary conditions along its four edges are given by

$$
\begin{gathered}
\text { at } x=0,0 \leqslant y \leqslant b \quad \tilde{u}=0 \text { and } \tilde{v}=0 \\
\text { at } 0 \leqslant x \leqslant l, y=0 \\
\qquad \tilde{t}_{x}=-\frac{2(2+3 v)}{v} f x^{5} \text { and } \tilde{t}_{y}=-\frac{2(4+3 v)}{(3+2 v)} c x^{5}
\end{gathered}
$$




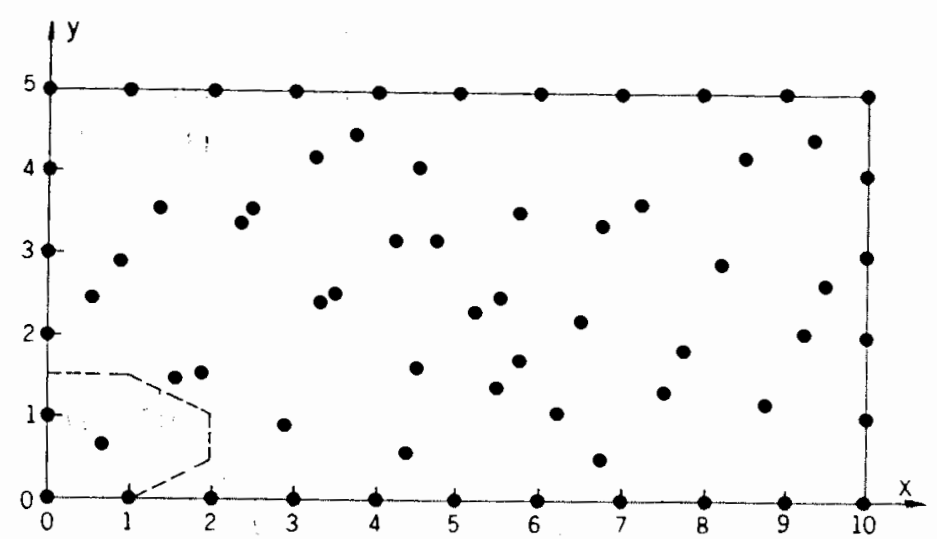

Figure 5. Schematic illustration of the computation domain of example I

at $0 \leqslant x \leqslant l, y=b$

$$
\begin{aligned}
\tilde{t}_{x}= & -\frac{2(2+3 v)}{v} f x^{5}-10 c b x^{4}+\frac{20(1+2 v)}{v} \int b^{2} x^{3} \\
& +\frac{20(2+v)}{(3+2 v)} c b^{3} x^{2}-10 f b^{4} x-\frac{2}{(3+2 v)} c b^{5}
\end{aligned}
$$

and

$$
\begin{aligned}
\tilde{t}_{y}= & -\frac{2(4+3 v)}{(3+2 v)} c x^{5}+\frac{10(2+3 v)}{v} f b x^{4}+20 c b^{2} x^{3} \\
& -\frac{20(1+2 v)}{v} f b^{3} x^{2}-\frac{10(2+v)}{(3+2 v)} c b^{4} x+2 f b^{5}
\end{aligned}
$$

at $x=l, 0 \leqslant y \leqslant b$

$$
\begin{aligned}
\hat{t}_{x}= & \frac{2}{v} \int y^{5}+\frac{10}{(3+2 v)} c l y^{4}+20 f l^{2} y^{3}-\frac{20(2+v)}{(3+2 v)} c l^{3} y^{2} \\
& -\frac{10(1+2 v)}{v} f l^{4} y+2 c l^{5} \\
\tilde{t}_{y}= & -\frac{2}{(3+2 v)} c y^{5}-10 f l y^{4}+\frac{20(2+v)}{(3+2 v)} c l^{2} y^{3} \\
& +\frac{20(1+2 v)}{v} \int 1^{3} y^{2}-10 c 1^{4} y-\frac{2(2+3 v)}{v} f 1^{5}
\end{aligned}
$$

where $l$ and $b$ are shown in Figure 5, $c$ and $f$ can be arbitrarily chosen.

The analytical solutions of this problem for the displacements $u(x, y)$ and $v(x, y)$ could be obtained using the polynomials method as described in Chapter 3 of Reference 34. Following 
their method, we derived the following analytical solution:

$$
\begin{aligned}
u(x, y)= & \frac{(v+1)^{2}}{(3+2 v) E} c x^{6}-\frac{2(3 v+1)(v+1)}{v E} f x^{5} y, \frac{10(v+1)^{2}}{(3+2 v) E} c x^{4} y^{2} \\
& +\frac{40 v(v+1)}{3 v E} f x^{3} y^{3}+\frac{5(v+1)^{2}}{(3+2 v) E} c x^{2} y^{4}+\frac{2\left(1-v^{2}\right)}{v E} f x y^{5}
\end{aligned}
$$

and

$$
\begin{aligned}
v(x, y)= & -\frac{4(v+1)(v+2)}{(3+2 v) E}\left(x^{5} y+\frac{10(v+1)^{2}}{v E} f x^{4} y^{2}+\frac{20(v+1)(v+3)}{3(3+2 v) E} c x^{3} y^{3}\right. \\
& -\frac{5(1+v)^{2}}{v E} \int x^{2} y^{4}-\frac{4(v+1)}{(3+2 v) E}\left(x y^{5}-\frac{(v+1)^{2}}{v E} f x^{6}\right.
\end{aligned}
$$

The analytical solutions for the stress components are

$$
\begin{aligned}
\sigma_{x}= & 2 c x^{5}-\frac{10(1+2 v)}{v} f x^{4} y-\frac{20(2+v)}{(3+2 v)} c x^{3} y^{2} \\
& +20 f x^{2} y^{3}+\frac{10}{(3+2 v)} c x y^{4}+\frac{2}{v} f y^{5} \\
\sigma_{y}= & \frac{-2(4+3 v)}{(3+2 v)} c x^{5}+\frac{10(2+3 v)}{v} f x^{4} y+20 c x^{3} y^{2} \\
& -\frac{20(1+2 v)}{v} f x^{2} y^{3}-\frac{10(2+v)}{(3+2 v)} c x y^{4}+2 f y^{5} \\
\tau_{x y}= & -\frac{2(2+3 v)}{v} f x^{5}-10 c x^{4} y+\frac{20(1+2 v)}{v} f x^{3} y^{2} \\
& +\frac{20(2+v)}{(3+2 v)}\left(x^{2} y^{3}-10 f x y^{4}-\frac{2}{(3+2 v)} c y^{5}\right.
\end{aligned}
$$

Note that the above solutions are for a case in which the body forces are assumed to be negligibly small.

The second example is shown in Figure 6. As a matter of fact it is a part cut out from the plate of the first example (see dashed line in Figure 5). Its boundary conditions were $\tilde{u}=0$ and $\tilde{v}$ at $(x=0$, $0 \leqslant y \leqslant b$ ). Along the other boundaries, the natural (traction) boundary conditions were calculated from the analytical solution given by equation (19).

This choice of the second example implies that its analytical solution is identical to that of the first example, i.e. equation (18). The second example was chosen in order to verify the applicability of the proposed method to inclined boundaries.

Both examples were solved numerically using the proposed method for $c=1$ and $f=1$. Sixty six nodal points were used in the first example and 52 in the second one. Their distributions in the appropriate computational domains are shown in Figures 5 and 6, respectively. Sixth-order Taylor series expansions were used in both solutions.

The numerical solutions of both examples were practically identical to the analytical ones. The only noticeable errors were due to the computer's round-off errors. These should not be surprising since the analytical solution [see equation (18)] is a sixth-order polynomial as are the Taylor series expansion approximations. 


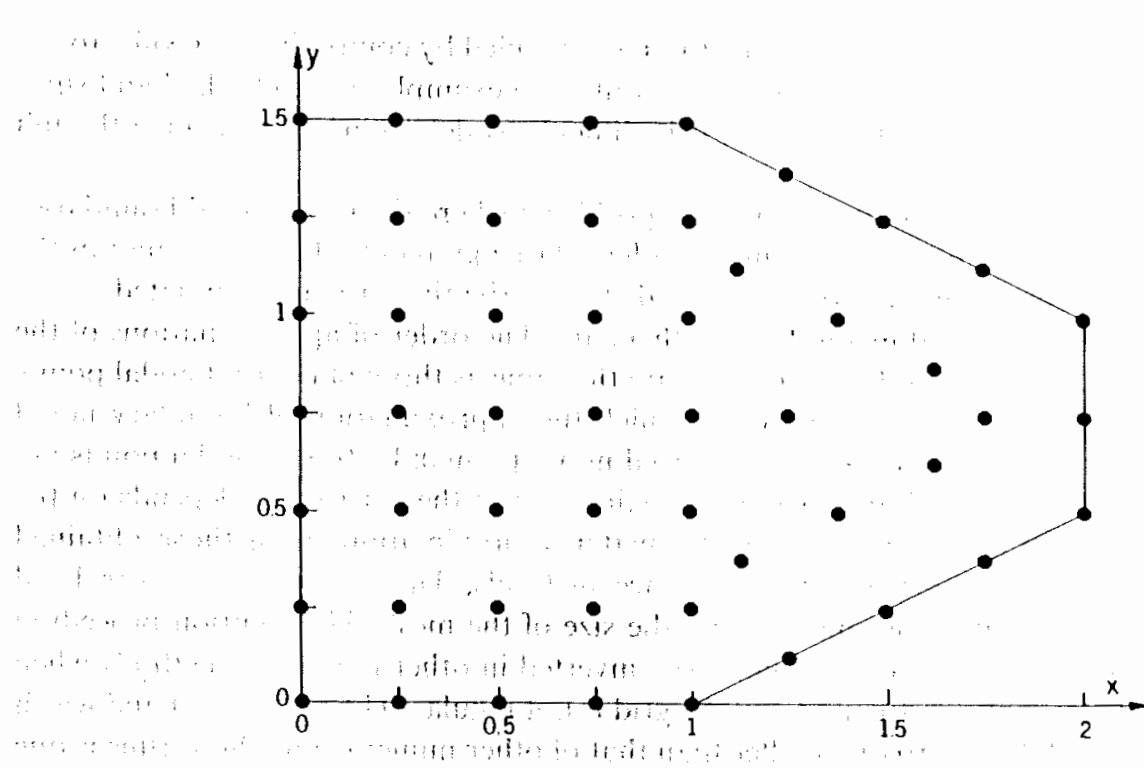

Figure 6. Schematic illustration of the computation domain of example 2

The use of standard finite difference methods or finite element methods (with eight-point elements) would inherently lead to much larger errors since their order of approximations are lower (usually 2 or 3 ). The use of sixth-order Taylor series expansion approximations with standard finite element or finite difference methods would result in very complex local schemes (28 neighbouring points for every element or nodal point).

The above-described two problems were solved using MATLAB which very conveniently treats algebraic manipulations with matrices. For this reason the $\mathrm{CPU}$ time required for a complete calculation was nót checked. The aim of the present paper is to present an idea for a possible improvement of the finite difference method and not to compete with computational time of 'other methods or compliter programs. A good programmer may adopt the proposed method to generate a very efficient code:

Consequently, it is probable that in spite of the improvements of our proposed method over other standard methods, there might be cases in which similar results can be achieved using the same computer time but less programming effort, by increasing the number of nodal points with standard methods. However, it is clear that there will be situations when the new proposed method will provide the only feasible method when computing power is limited. In addition 10 solving engineering problems, the proposed method can be used as a tool for comparison between other numerical methods

It should be noted here that although straight and oblique boundaries were used in the presented two examples, the method can be used with curved boundaries as well. Owing to the difficulty in differentiating the' natural '(traction) boundary condition equations along curved boundaries such an example was not demonstrated here.

\subsection{CONCLUSIONS}

A new idea for the numerical solution of partial differential equations of equilibrium problems was forwarded and presented. The idea is based on differentiation of both the governing and the 
boundary condition equations. The proposed method was verified by comparing its results to the analytical results of two problems in elasticity. Since only two examples were checked and since the idea, to the best of our knowledge, is new, it is difficult to determine at this stage the full applicability of the proposed method.

The method would be most useful in equilibrium problems where the geometrical boundaries are curved and the boundary conditions along them have large gradients. This is because in the proposed method both the boundary conditions and their derivatives are approximated.

The advantages of the proposed method over others are: The order of approximations of the solution at nodal points located on the boundaries are the same as those of internal nodal points (in contrast to other finite difference methods in which the approximation of boundary nodal points are one order of magnitude lower than internal nodal points); Fictilious nodal points are not required; The numerical algorithm fer the approximation of the derivatives depends on the entire differential equation and therefore provides better approximations than those obtained from the fix numerical algorithms of finite difference methods; The method uses more local equations and hence the solution depends less on the size of the mesh; The solution procedure requires the inversion of smaller matrices than those inverted in other numerical methods when the same order of approximations are used and the grid is not regular; The size of the band width of the finally generated global matrix is smaller than that of other numerical methods (this is due to the use of less neighbouring points, i.e. smaller local schemes). Consequently, the improvement of the proposed solution procedure over other procedures becomes more and more pronounced as the number of the nodal points increases.

The disadvantages of the proposed method in relation to others are: It is relativcly a cumbersome method in its implementation. Prior to its application it requres a large volume of analytical calculations, i.e. successive differentiation of both the governing and boundary condition equations. It requires a manual selection of the local schemes (i.e. templates) for each nodal point. (Note that this can be overcome by appropriate programming which will account for the criterion suggested to avoid singularities and ill-conditioning).

Because the new method describes a method for solving equilibrium problems when the spatial region is irregular in shape, and provides consistent accuracy everywhere, it will be useful to many researchers and engineers.

Finally, it should be noted that the present method (idea) has not been applied yet to non-lincar differential equations where convergence must be guaranteed.

\section{REFERENCES}

1. O. C. Zienkiewicz, 'The finite element method: From intuition to generality', Appl. Mech. Rev., 23, 249-256 (1970).

2. R. V. Southwell, Relaxation Methods in Engineering Science, Oxford University Press, Oxford, 1940.

3. R. V. Southwell, Relaxation Methods in Theoretical Physics, Clarenden, Oxford, 1946.

4. G. E. Forsyth and W. R. Wasow, Finite Difference Methods for Partial Differential Equations, Wiley, New York, 1960.

5. C. T. Wang, Applied Elasticity, McGraw-Hill, New York, 1953.

6. L. Collatz, The Numerical Treatment of Differential Equations, 3rd edn., Springer, Berlin, 1960.

7. R. S. Varga, Matrix Iterative Analysis, Prentice-Hall, New Jersey, 1960.

8. D.S. Griffin and R. B: Kellogg, 'A numerical solution for axjally symmetric and plane elasticity problems, Int, J. Solids Struct., 3, 781 (1967).

9. S. Utka, 'Stiffness matrices for thin triangular elements of nonzero Gaussian curvature', AIAA J., 5, 1659 1667 (1967)

10. K. S. Havner and E. L. Stanton, 'On energy-derived difference equations in thermal stress problems', J. Franklin Inst., 284, 127 (1967).

11. K. S. Havner, 'On convergence of iterative methods in plastic strain analysis', Int. J. Solids Struct., 4, 491 (1968).

12. D. E. Johnson, 'A difference-based variational method for shells', Int. J. Solids Struct., 6, 699-724 (1970).

13. P. S. Jensen, 'Finite' difference technique for variable grids', J. Comp. Struct., 2, 17-29 (1972).

14. R. H. MacNeal, 'An asymmetrical finite difference network', Quart. Appl. Math., 11, 295-310 (1953) 
15. R. B. Kellogg, 'A finite difference approximation to the diffusion equation in hexagonal geometry', Comb. Eng., Inc. Rept. MPC-21, Windsor, CT., (1959).

16. R. B. Kellogg, 'A Ritz finite difference approximation to the neutron diffusion equation', Bettis Tech., Rev., WAPD-BT-31, 51-58, 1964.

17. R. B. Kellogg, 'Difference equations in a mesh arising from a general triangulation', Math. Comp., 18, 203 210(1964).

18. R. B. Kellogg, 'The errors for finite difference approximations to the diffusion equation', Comb. Eng.. Inc. Rept. MPC-24, Windsor, CT, (1960).

19. A. M. Winslow, 'Numerical solution of the quasi-linear Poisson equation in a non-uniform triangular mesh', J. Comp. Phys., 2, $72-149$ (1966).

20. W. H. Chu, 'Development of a general finile difference approximation for a general domain. Part I: Machine transformation', J. Comp. Phys., 8, 392-408 (1971).

21. W. H. Chu, 'Low-gravity fuel sloshing in an arbitrary axisymmetric rigid tank', ASME Paper 70-APM-EEE, J. Appl. Mech., 37, 828-837 (1970).

22. W. H. Frey, 'Flexible finite-difference stencils from iso-parametric finite elements', Int. j. numer, methods eng., 11 , $1653-1665$ (1977).

23. N. Perrone and R. Kao, 'A general finite difference method for arbitrary meshes', J. Comp. Struct., 5, 45-58 (1975).

24. T. Liszka and J. Orkisz, "The finite difference method at arbitrary irregular grids and its application in applied mechanics', Comp. Struct., 11, 83-95 (1980).

25. B. J. Noye and R. J. Arnold, 'Accurate finite different approximations for the Neumann condition on a curved boundary', Appl. Math. Modelling, 14, 1-13, (1990).

26. J. O. Dow, M. S. Jones and S. A. Harwood, 'A new approach to boundary modeling for finite difference applications in solid mechanics', Int. j. numer. methods eng., 30, 99-113 (1990).

27. J. O. Dow and J. L. Hardaway, 'Validation of finite difference boundary condition models for solid mechanics applications' AlA $A J ., 30(7), 1864-1869$ (1992).

28. J. O. Dow, T. H. Ho and H. D. Cabiness, 'Generalized finite element evaluation procedure', J. Struct. Div. ASCE, 111, $435-452(1985)$

29. J. O. Dow, Z. W. Su, C. C. Feng and C. Bodley, 'Equivalent continuum representation of structures composed of repeated elements', AlAA J., 23(10), I564-1569 (1985).

30. J. O. Dow, H. D. Cabiness and T. H. Ho, 'Linear strain element with curved edges', J. Struct. Div, ASCE, 112, 692-708 (1986).

31. J. O. Dow and D. E. Byrd, 'The identification and elimination of artificial stiffening errors in finite elements', $\ln t . j$. numer. methods eng., 26, 743-762 (1988).

32. E. Kochavi, R. Segev and Y. Yomdin, 'Numerical solution ef field problems by nonconforming Taylor discrelization', Appl. Math. Modelling, 15, 152-157 (1991).

33. J. N. Reddy, An Introduction to the Finite Element Method, McGraw-Hill, New York, 1984.

34. S. P. Timoshenko and J. N. Goodier, Theory of Elasticity, 3rd edn., McGraw, Singapore, 1970. 